Article

\title{
Spectral Galerkin Approximation of Space Fractional Optimal Control Problem with Integral State Constraint
}

\author{
Fangyuan Wang, Xiaodi Li and Zhaojie Zhou *
}

check for

updates

Citation: Wang, F.; Li, X.; Zhou, Z. Spectral Galerkin Approximation of Space Fractional Optimal Control Problem with Integral State Constraint. Fractal Fract. 2021, 5, 102. https://doi.org/

10.3390/fractalfract5030102]

Academic Editors: Vassili Kolokoltsov and David Kubanek

Received: 28 June 2021

Accepted: 8 August 2021

Published: 24 August 2021

Publisher's Note: MDPI stays neutral with regard to jurisdictional claims in published maps and institutional affiliations.

Copyright: (c) 2021 by the authors. Licensee MDPI, Basel, Switzerland. This article is an open access article distributed under the terms and conditions of the Creative Commons Attribution (CC BY) license (https:// creativecommons.org/licenses/by/ $4.0 /)$.
School of Mathematics and Statistics, Shandong Normal University, Jinan 250014, China; wfy_sdnu@163.com (F.W.); 1xd@sdnu.edu.cn (X.L.)

* Correspondence: zhouzhaojie@sdnu.edu.cn

Abstract: In this paper spectral Galerkin approximation of optimal control problem governed by fractional advection diffusion reaction equation with integral state constraint is investigated. First order optimal condition of the control problem is discussed. Weighted Jacobi polynomials are used to approximate the state and adjoint state. A priori error estimates for control, state, adjoint state and Lagrangian multiplier are derived. Numerical experiment is carried out to illustrate the theoretical findings.

Keywords: spectral Galerkin method; optimal control problem; state constraint; weighted Jacobi polynomials; a priori error estimate

\section{Introduction}

The aim of this paper is to develop a spectral Galerkin approximation of the following optimal control problem governed by fractional advection diffusion reaction equation:

$$
\min _{(y(u), u) \in G_{a d} \times L^{2}(\Omega)} J(y, u):=\frac{1}{2} \int_{\Omega}\left(y(x)-y_{d}(x)\right)^{2} d x+\frac{\gamma}{2} \int_{\Omega} u^{2}(x) d x
$$

subject to

$$
\left\{\begin{aligned}
(-\Delta)^{\frac{\alpha}{2}} y(x)+\mu_{1} D y(x)+\mu_{2} y(x) & =f+u(x), & & x \in \Omega, \\
y(x) & =0, & & x \in \Omega^{c}
\end{aligned}\right.
$$

and the state constraint

$$
G_{a d}=\left\{v \in L^{1}(\Omega) \mid \int_{\Omega} v d x \leq d\right\},
$$

where $\Omega=(-1,1), \Omega^{c}=\mathbb{R} \backslash \Omega$, and $D$ is the first-order derivative with respect to $x$. Here $\mu_{1} \neq 0$ is a constant and $\mu_{2} \geq 0 . f(x)$ is a given function and $y_{d}$ is the desired state. $(-\triangle)^{\frac{\alpha}{2}}$ denotes the fractional Laplacian operator defined in integral form:

$$
(-\Delta)^{\frac{\alpha}{2}} y(x)=c_{1, \alpha} \int_{\mathbb{R}} \frac{y(x)-y(\xi)}{|x-\xi|^{1+\alpha}} d \xi, \quad c_{1, \alpha}=\frac{2^{\alpha} \Gamma((\alpha+1) / 2)}{\pi^{1 / 2}|\Gamma(-\alpha / 2)|}, \alpha \in(1,2) .
$$

Fractional calculus has wide applications in many fields including anomalous diffusion processes [1-3], control theory [4-8], fractional-order neural networks [9], biomedical applications [10,11], mechatronics [12,13], etc. In the past decades lots of works [14-19] are devoted to develop numerical methods or algorithms for fractional differential equations. In recent years optimal control problems governed by different types of fractional differential equations have attracted increasing attentions [20-30].

The abnormal diffusion phenomenon widely exists in our real world, for example, the pollutant transport in groundwater, where the solutes moving through aquifers do not generally follow a classical second-order Fickian diffusion equation [1,2]. The heavy tail behavior of the transport processes can be accurately described by Levy distribution. This 
can be viewed as a probability description of fractional advection diffusion equations. The plume spreads faster than a traditional Brownian motion due to the self-similarity. The traditional dispersion equation would seriously underestimate the risk of downstream contamination if the plume represent a pollutant heading to a drinking water well. The stable density that solves the fractional diffusion equation can capture the super-diffusive spreading observed in the data. Motivated by these facts, in this paper we mainly focus on the optimal control problem governed by a fractional advection diffusion equation.

To achieve higher-order convergence, spectral methods based on weighted polynomials (the product of weighted functions and polynomials) have been developed to solve fractional differential equations [31-33], which naturally accommodate the weak singularity of fractional derivative at the endpoint. A spectral Galerkin approximation of optimal control problem governed by fractional equations with control constraint is firstly investigated in [34,35], where the weighted Jacobi polynomials are used to approximate the state variable and the adjoint state variable. As an extension in the present work we propose a spectral Galerkin approximation scheme for optimal control problem governed by fractional advection diffusion reaction equation under the constraints of state integration. Our model is general in that it includes advection, reaction and diffusion terms, which are seldom studied in the literature, especially for the optimal control of the corresponding state integral constraints. We proved a priori error analysis for state variable, adjoint state variable, control variable, and Lagrangian multiplier, and the boundary singularities of the solutions are considered in the convergence estimates, which provides a characterization for the space-fractional problems and distinguishes this paper from many existing works assuming the solutions to be sufficiently smooth. Finally numerical example is given to illustrate the theoretical result.

The paper is organized as follows. In Section 2, we recall on some preliminary knowledge and derive the continuous first-order optimality condition. In Section 3, we construct a spectral Galerkin discrete scheme for optimal control problem, where weighted Jacobi polynomials are used. Then a discrete first-order optimality condition is deduced, and a priori error estimates of state variable, adjoint variable, control variable, and Lagrangian multiplier are proved. In Section 4, numerical example is given to confirm our theoretical findings.

\section{Preliminary Knowledge}

In this section, we begin with a brief review of the definitions and properties of weighed Sobolev spaces, fractional Laplacian operator, and Jacobi polynomials. Then we derive the first order optimality condition.

\subsection{Weighed Sobolev Spaces and Jacobi Polynomials}

Denote by $L_{\omega^{\alpha / 2}}^{2}$ the space with the inner product and norm defined by

$$
(u, v)_{\omega^{\alpha / 2}}=\int_{\Omega} u v \omega^{\alpha / 2} d x, \quad\|u\|_{\omega^{\alpha / 2}}=(u, u)_{\omega^{\alpha / 2}}^{1 / 2}, \forall u, v \in L_{\omega^{\alpha / 2}}^{2}
$$

where $\omega^{\alpha / 2}(x)=\left(1-x^{2}\right)^{\alpha / 2}$ is a weight function.

We denote by $\mathbb{P}_{\mathbb{N}}$ the set of Jacobi polynomials of degree at most $N$. The Jacobi polynomials $P_{n}^{\alpha / 2}$ in $\mathbb{P}_{\mathbb{N}}$ are mutually orthogonal as follows

$$
\int_{\Omega} \omega^{\alpha / 2}(x) P_{n}^{\alpha / 2}(x) P_{m}^{\alpha / 2}(x) d x=h_{n}^{\alpha / 2} \delta_{n m}, \delta_{n m} \text { is the Dirac delta symbol }
$$

and

$$
h_{n}^{\alpha / 2}=\frac{2^{\alpha+1}(\Gamma(n+\alpha / 2+1))^{2}}{(2 n+\alpha+1) \Gamma(n+\alpha+1) \Gamma(n+1)} .
$$

Lemma 1 (See [32]). The following relation holds for the Jacobi polynomials $P_{n}^{\alpha / 2}(x)$ 


$$
(-\Delta)^{\frac{\alpha}{2}}\left[\omega^{\alpha / 2} P_{n}^{\alpha / 2}(x)\right]=g_{n}^{\alpha} P_{n}^{\alpha / 2}(x), \quad g_{n}^{\alpha}=\frac{\Gamma(\alpha+n+1)}{n !} .
$$

Lemma 2. The first order derivative of the Jacobi polynomials $P_{n}^{\alpha / 2}(x)$ satisfies

$$
D\left(\omega^{\alpha / 2} P_{n-1}^{\alpha / 2}\right)=-2 n\left(1-x^{2}\right)^{\alpha / 2-1} P_{n}^{\alpha / 2-1} .
$$

To incorporate singularities at the endpoints, we use the following non-uniformly Jacobi-weighted Sobolev space (see [36,37])

$$
B_{\omega^{\alpha / 2}}^{s}=\left\{u \mid \partial^{k} u \in L_{\omega^{\alpha / 2+k}}^{2}, k=0,1, \ldots s\right\}, \mathrm{s} \text { is a nonnegative integer, }
$$

which is equipped with the following norm and seminorm

$$
\|u\|_{B_{\omega^{\alpha} / 2}^{s}}=\left(\sum_{k=0}^{s}|u|_{B_{\omega^{\alpha / 2}}^{k}}\right)^{1 / 2}, \quad|u|_{B_{\omega^{\alpha / 2}}^{k}}=\left\|\partial^{k} u\right\|_{\omega^{\alpha / 2+k}} .
$$

When $s$ is not an integer, the space is defined by interpolation (see [36,37]).

\subsection{Properties of the Fractional Laplacian Operations}

Lemma 3 (See [32]). Assume that $u, v$ vanish outside of $\Omega \subseteq \mathbb{R}$ almost everywhere. Then it holds that

$$
\begin{aligned}
\int_{\Omega} v(-\Delta)^{\frac{\alpha}{2}} u(x) d x= & \frac{c_{1, \alpha}}{2} \int_{\Omega} \int_{\Omega} \frac{(u(x)-u(y))(v(x)-v(y))}{|x-y|^{1+\alpha}} d y d x \\
& +\int_{\Omega} u(x) v(x) \rho(x) d x,
\end{aligned}
$$

when all the integrals are well-defined. Here $\rho(x)$ is defined as follows

$$
\rho(x)=c_{1, \alpha} \int_{\Omega^{c}} \frac{1}{|x-y|^{1+\alpha}} d y=\frac{c_{1, \alpha}}{\alpha}\left((1+x)^{-\alpha}+(1-x)^{-\alpha}\right) \geq \frac{c_{1, \alpha}}{\alpha} \omega^{-\alpha} .
$$

Lemma 4. By Lemma 1, we can get

$$
\left((-\Delta)^{\frac{\alpha}{2}} \eta+\mu_{2} \eta, \eta\right)=\frac{C_{1}}{2}|\eta|_{H^{\alpha / 2}}^{2}+\mu_{2}\|\eta\|^{2}+\left\|\eta \rho^{1 / 2}\right\|^{2} .
$$

Here $C_{1}>0$ and following [32] we know that $\rho(x) \geq \frac{C_{1}}{\alpha} \omega^{-\alpha}$. Then we have

$$
\left((-\Delta)^{\frac{\alpha}{2}} \eta+\mu_{2} \eta, \eta\right) \geq \frac{C_{1}}{2}|\eta|_{H^{\alpha / 2}}^{2}+\mu_{2}\|\eta\|^{2}+\frac{C_{1}}{\alpha}\|\eta\|_{\omega^{-\alpha / 2}}^{2} .
$$

\subsection{First-Order Optimality Condition}

Theorem 1. Assume that $(y, u)$ is the solution to optimal control problem (1) and (2). Then the following first-order optimality condition holds

$$
\left\{\begin{aligned}
(-\Delta)^{\frac{\alpha}{2}} y+\mu_{1} D y+\mu_{2} y & =f+u \\
(-\Delta)^{\frac{\alpha}{2}} z-\mu_{1} D z+\mu_{2} z & =y-y_{d}+\mu \\
(\mu, v-y) & \leq 0, \forall v \in G_{a d} \\
z+\gamma u & =0
\end{aligned}\right.
$$

Here

$$
\mu= \begin{cases}0, & \text { if } \int_{\Omega} y d x<d, \\ \text { constant } \geq 0, & \text { if } \int_{\Omega} y d x=d,\end{cases}
$$


$\mu_{1} \neq 0$ is a constant and $\mu_{2} \geq 0$.

Proof. To derive the first order optimality system, we set $F(u):=\int_{\Omega} y(u) d x-d$, where $y(u)$ is the solution of the state equation associated to $u$. Then according to $[38,39]$ there exist a real number $\mu \geq 0$ such that

$$
\mu F(u)=0
$$

and

$$
L_{u}^{\prime}(u)(v-u)=0, \forall v \in U_{a d} .
$$

Here $L(u, \mu)=\hat{J}(u)+\mu F(u)$ denote the Lagrangian functional with $\mu$ being the Lagrangian multiplier.

By simple calculation we have

$$
\begin{aligned}
\hat{J}^{\prime}(u)(v-u) & =\lim _{\lambda \rightarrow 0} \frac{\hat{J}(u+\lambda(v-u))-\hat{J}(u)}{\lambda} \\
& =\int_{\Omega}\left(y(u)-y_{d}\right)\left[y^{\prime}(u)(v-u)\right] d x+\gamma \int_{\Omega} u(v-u) d x
\end{aligned}
$$

and

$$
\begin{aligned}
\mu F^{\prime}(u)(v-u) & =\mu \lim _{\lambda \rightarrow 0} \frac{F(u+\lambda(v-u))-F(u)}{\lambda} \\
& =\int_{\Omega} \mu y^{\prime}(u)(v-u) d x .
\end{aligned}
$$

Then we obtain

$$
\begin{aligned}
L_{u}^{\prime}(u)(v-u)= & \int_{\Omega}\left(y(u)-y_{d}\right)\left[y^{\prime}(u)(v-u)\right] d x+\gamma \int_{\Omega} u(v-u) d x \\
& +\mu \int_{\Omega} y^{\prime}(u)(v-u) d x=0, \forall v \in U_{a d} .
\end{aligned}
$$

Let $q=y^{\prime}(u)(v-u)$. It follows from the state equation that

$$
\left\{\begin{aligned}
(-\Delta)^{\frac{\alpha}{2}} q+\mu_{1} D q+\mu_{2} q & =v-u, \quad x \in \Omega, \\
q & =0, \quad x \in \Omega^{c} .
\end{aligned}\right.
$$

Then we introduce the adjoint state equation:

$$
\left\{\begin{aligned}
(-\Delta)^{\frac{\alpha}{2}} z(x)-\mu_{1} D z(x)+\mu_{2} z(x) & =y(x)-y_{d}+\mu, & x \in \Omega, \\
z(x) & =0, & x \in \Omega^{c},
\end{aligned}\right.
$$

By integration-by-parts, we deduce

$$
\begin{aligned}
& \int_{\Omega}\left(y(u)-y_{d}+\mu\right)\left[y^{\prime}(u)(v-u)\right] d x \\
& =\int_{\Omega}\left((-\Delta)^{\frac{\alpha}{2}} z(x)-\mu_{1} D z(x)+\mu_{2} z(x)\right) q(x) d x \\
& =\int_{\Omega}\left((-\Delta)^{\frac{\alpha}{2}} q(x)+\mu_{1} D q(x)+\mu_{2} q(x)\right) z(x) d x \\
& =\int_{\Omega} z(v-u) d x .
\end{aligned}
$$

Combing the above equations leads to 


$$
L_{u}^{\prime}(u)(v-u)=\int_{\Omega}(\gamma u+z(x))(v-u) d x=0, \forall v \in G_{a d} .
$$

This implies (8). Note that for $v \in G_{a d}$

$$
\begin{aligned}
0=\mu F(u) & =\mu\left(\int_{\Omega} y(u) d x-d\right) \\
& =\mu\left(\int_{\Omega}(y(u)-v) d x\right)+\mu\left(\int_{\Omega} v d x-d\right) .
\end{aligned}
$$

This implies for $v \in G_{a d}$

$$
\mu(1, v-y(u))=\mu\left(\int_{\Omega} v d x-d\right) \leq 0 .
$$

Remark 1. From (8) we can further derive that

$$
\mu= \begin{cases}0, & \text { if } \int_{\Omega} y d x<d, \\ \text { constant } \geq 0, & \text { if } \int_{\Omega} y d x=d .\end{cases}
$$

\section{Spectral Galerkin Approximation}

Define

$$
V_{N}=\omega^{\alpha / 2} \mathbb{P}_{\mathbb{N}}=\operatorname{Span}\left\{\phi_{0}, \phi_{1}, \ldots, \phi_{N}\right\},
$$

where $\phi_{k}(x)=\omega^{\alpha / 2} P_{k}^{\alpha / 2}(x)$ for $0 \leq k \leq N$. Let $\mathcal{V}_{N}=V_{N} \cap G_{a d}$. The spectral Galerkin method for optimal control problem (1) and (2) is to find $\left(y_{N}, u_{N}\right)$ satisfying

$$
\min _{\left(y_{N}, u_{N}\right) \in \mathcal{V}_{N} \times V_{N}} J\left(y_{N}, u_{N}\right):=\frac{1}{2} \int_{\Omega}\left(y_{N}(x)-y_{d}(x)\right)^{2} d x+\frac{\gamma}{2} \int_{\Omega} u_{N}^{2}(x) d x
$$

subject to

$$
\left((-\Delta)^{\frac{\alpha}{2}} y_{N}+\mu_{1} D y_{N}+\mu_{2} y_{N}, v_{N}\right)=\left(f+u_{N}, v_{N}\right), \forall v_{N} \in V_{N} .
$$

In a similar way to continuous case we have the following discrete optimality condition

$$
\left\{\begin{array}{clrl}
\left((-\Delta)^{\frac{\alpha}{2}} y_{N}+\mu_{1} D y_{N}+\mu_{2} y_{N}, v_{N}\right) & =\left(f+u_{N}, v_{N}\right), & & \forall v_{N} \in V_{N} \\
\left((-\Delta)^{\frac{\alpha}{2}} z_{N}-\mu_{1} D z_{N}+\mu_{2} z_{N}, v_{N}\right)=\left(y_{N}-y_{d}+\mu_{N}, v_{N}\right), & & \forall v_{N} \in V_{N} \\
\mu_{N}\left(1, v_{N}-y_{N}\right) \leq 0, & & \forall v_{N} \in \mathcal{V}_{N} \\
z_{N}+\gamma u_{N}=0 . & &
\end{array}\right.
$$

Here

$$
\mu_{N}=\left\{\begin{array}{cc}
0, & \text { if } \int_{\Omega} y_{N} d x<d, \\
\text { constant } \geq 0, & \text { if } \int_{\Omega} y_{N} d x=d .
\end{array}\right.
$$

To achieve the error estimate, we need to introduce the following auxiliary problems: 


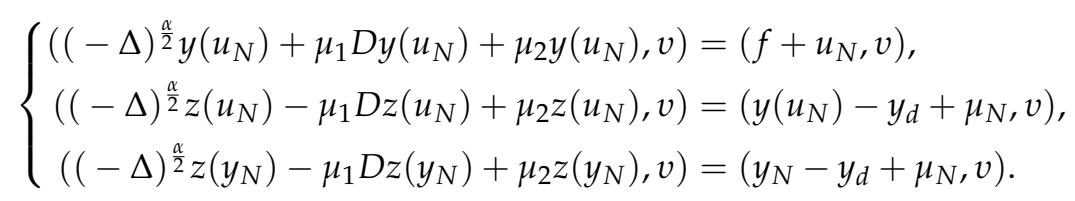

Lemma 5 (see [40]). Assume that $\eta$ and $\eta_{N}$ are solutions of state equation and its discrete counterpart, respectively. Let $\omega^{-\alpha / 2} \eta \in B_{\omega^{\alpha / 2}}^{s}$. Then for $s \geq \alpha / 2$ we have

$$
\left\|\eta-\eta_{N}\right\|_{\omega^{-\alpha / 2}}+N^{-\alpha / 2}\left\|\eta-\eta_{N}\right\|_{H^{\alpha / 2}} \leq C N^{-s}\left|\omega^{-\alpha / 2} \eta\right|_{B^{\alpha} / 2} .
$$

Lemma 6. Assume that $(y, z, u, \mu)$ and $\left(y_{N}, z_{N}, u_{N}, \mu_{N}\right)$ are the solutions of optimality conditions (8) and (11) respectively. Suppose that $\omega^{-\alpha / 2} y, \omega^{-\alpha / 2} z \in B_{\omega^{\alpha / 2}}^{s}, s \geq \alpha / 2$. Then the following error estimates hold

$$
\begin{aligned}
& \left\|y-y_{N}\right\|_{\omega^{-\alpha / 2}}+\left\|z-z_{N}\right\|_{\omega^{-\alpha / 2}} \leq C N^{-s}+C\left(\left\|u-u_{N}\right\|+\left|\mu-\mu_{N}\right|\right), \\
& \left\|y-y_{N}\right\|_{H^{\alpha / 2}}+\left\|z-z_{N}\right\|_{H^{\alpha / 2}} \leq C N^{\alpha / 2-s}+C\left(\left\|u-u_{N}\right\|+\left|\mu-\mu_{N}\right|\right) .
\end{aligned}
$$

Proof. Note that $y_{N}$ and $z_{N}$ are the spectral Galerkin approximation of $y\left(u_{N}\right)$ and $z\left(y_{N}\right)$, respectively. Therefore, by Lemma 5 we have

$$
\begin{aligned}
\left\|y_{N}-y\left(u_{N}\right)\right\|_{\omega^{-\alpha / 2}}+N^{-\alpha / 2}\left\|y_{N}-y\left(u_{N}\right)\right\|_{H^{\alpha / 2}} & \leq C N^{-s}, \\
\left\|z_{N}-z\left(y_{N}\right)\right\|_{\omega^{-\alpha / 2}}+N^{-\alpha / 2}\left\|z_{N}-z\left(y_{N}\right)\right\|_{H^{\alpha / 2}} & \leq C N^{-s} .
\end{aligned}
$$

By (8) and (13) we have

$$
\begin{aligned}
& \left((-\Delta)^{\frac{\alpha}{2}}\left(y-y\left(u_{N}\right)\right)+\mu_{1} D\left(y-y\left(u_{N}\right)\right)+\mu_{2}\left(y-y\left(u_{N}\right)\right), v\right) \\
& =\left(u-u_{N}, v\right) .
\end{aligned}
$$

Choosing $v=y-y\left(u_{N}\right)$ leads to

$$
\begin{aligned}
& \left((-\Delta)^{\frac{\alpha}{2}}\left(y-y\left(u_{N}\right)\right)+\mu_{1} D\left(y-y\left(u_{N}\right)\right)+\mu_{2}\left(y-y\left(u_{N}\right)\right), y-y\left(u_{N}\right)\right) \\
= & \left(u-u_{N}, y-y\left(u_{N}\right)\right) .
\end{aligned}
$$

By integration-by-parts, we have

$$
\mu_{1}\left(D\left(y-y\left(u_{N}\right)\right),\left(y-y\left(u_{N}\right)\right)\right)=-\mu_{1}\left(y-y\left(u_{N}\right), D\left(y-y\left(u_{N}\right)\right)\right) .
$$

This yields

$$
\mu_{1}\left(D\left(y-y\left(u_{N}\right)\right),\left(y-y\left(u_{N}\right)\right)\right)=0 .
$$

By (7), we can derive

$$
\begin{aligned}
& \left((-\Delta)^{\frac{\alpha}{2}}\left(y-y\left(u_{N}\right)\right)+\mu_{2}\left(y-y\left(u_{N}\right)\right), y-y\left(u_{N}\right)\right) \\
& \geq \frac{C_{1}}{2}\left|y-y\left(u_{N}\right)\right|_{H^{\alpha / 2}}^{2}+\mu_{2}\left\|y-y\left(u_{N}\right)\right\|^{2}+\frac{C_{1}}{\alpha}\left\|y-y\left(u_{N}\right)\right\|_{\omega^{-\alpha / 2}}^{2} .
\end{aligned}
$$

Note that

$$
\left(u-u_{N}, y-y\left(u_{N}\right)\right) \leq\left\|y-y\left(u_{N}\right)\right\|_{\omega^{-\alpha / 2}}\left\|u-u_{N}\right\|_{\omega^{\alpha / 2}} .
$$

Then using the Young inequality we further have

$$
\begin{aligned}
& \quad\left|y-y\left(u_{N}\right)\right|_{H^{\alpha / 2}}+\left\|y-y\left(u_{N}\right)\right\|+\left\|y-y\left(u_{N}\right)\right\|_{\omega^{-\alpha / 2}} \\
& \leq C\left\|u-u_{N}\right\|_{\omega^{\alpha / 2}} \leq C\left\|u-u_{N}\right\| .
\end{aligned}
$$


By (8) and (13) we have

$$
\begin{aligned}
& \left((-\Delta)^{\frac{\alpha}{2}}\left(z-z\left(y_{N}\right)\right)-\mu_{1} D\left(z-z\left(y_{N}\right)\right)+\mu_{2}\left(z-z\left(y_{N}\right)\right), v\right) \\
= & \left(y-y_{N}, v\right)+\left(\mu-\mu_{N}, v\right) .
\end{aligned}
$$

Further, by setting $v=z-z\left(y_{N}\right)$ we obtain

$$
\begin{aligned}
& \left((-\Delta)^{\frac{\alpha}{2}}\left(z-z\left(y_{N}\right)\right)-\mu_{1} D\left(z-z\left(y_{N}\right)\right)+\mu_{2}\left(z-z\left(y_{N}\right)\right), z-z\left(y_{N}\right)\right) \\
= & \left(y-y_{N}, z-z\left(y_{N}\right)\right)+\left(\mu-\mu_{N}, z-z\left(y_{N}\right)\right) .
\end{aligned}
$$

In a similar way to state variable, using Lemma 5 we can deduce

$$
\begin{aligned}
& \left|z-z\left(y_{N}\right)\right|_{H^{\alpha / 2}}+\left\|z-z\left(y_{N}\right)\right\|+\left\|z-z\left(y_{N}\right)\right\|_{\omega^{-\alpha / 2}} \\
\leq & C\left\|y-y_{N}\right\|+C\left|\mu-\mu_{N}\right| \\
\leq & C\left\|y-y\left(u_{N}\right)+y\left(u_{N}\right)-y_{N}\right\|+C\left|\mu-\mu_{N}\right| \\
\leq & C\left(N^{-s}+\left\|u-u_{N}\right\|+\left|\mu-\mu_{N}\right|\right) .
\end{aligned}
$$

Combining (14)-(16) yields the final results.

Note that the estimate of the state and the adjoint state depends on the estimate of the $\| u-u_{N}||$ and $\left|\mu-\mu_{N}\right|$. In the following we are going to estimate $\left|\mu-\mu_{N}\right|$ first.

Lemma 7. Let $(y, z, u, \mu)$ and $\left(y_{N}, z_{N}, u_{N}, \mu_{N}\right)$ be the solutions of (8) and the discrete counterpart, respectively. Then the following estimate holds

$$
\left|\mu-\mu_{N}\right| \leq C\left(N^{-s}+\left\|u-u_{N}\right\|\right) .
$$

Proof. Note that

$$
\begin{aligned}
& \left((-\Delta)^{\frac{\alpha}{2}}\left(z-z\left(u_{N}\right)\right)-\mu_{1} D\left(z-z\left(u_{N}\right)\right)+\mu_{2}\left(z-z\left(u_{N}\right)\right), v\right) \\
= & \left(y-y\left(u_{N}\right), v\right)+\left(\mu-\mu_{N}, v\right) .
\end{aligned}
$$

Choosing $v=w \in C_{0}^{\infty}$ with $\frac{1}{|\Omega|} \int_{\Omega} w d x=1$ and $\|w\|_{H^{1}(\Omega)} \leq C$ leads to

$$
\begin{aligned}
\left(\mu-\mu_{N}, w\right)= & \left((-\Delta)^{\frac{\alpha}{2}}\left(z-z\left(u_{N}\right)\right)+\mu_{2}\left(z-z\left(u_{N}\right)\right), w\right)+\mu_{1}\left(z-z\left(u_{N}\right), D w\right) \\
& -\left(y-y\left(u_{N}\right), w\right) .
\end{aligned}
$$

Then by (18) we can get

$$
\begin{aligned}
\left|\mu-\mu_{N}\right| & \leq C\left(\left|z-z\left(u_{N}\right)\right|_{H^{\alpha / 2}}+\left\|z-z\left(u_{N}\right)\right\|+\left\|y-y\left(u_{N}\right)\right\|\right) \\
& \leq C\left(\left|z-z\left(u_{N}\right)\right|_{H^{\alpha / 2}}+\left\|z-z\left(u_{N}\right)\right\|+\left\|u-u_{N}\right\|\right) .
\end{aligned}
$$

Set $Z=\frac{1}{|\Omega|} \int_{\Omega}\left(z-z\left(u_{N}\right)\right) d x$. By setting $v=z-z\left(u_{N}\right)-Z w$ in (17) we have

$$
\begin{gathered}
\left((-\Delta)^{\frac{\alpha}{2}}\left(z-z\left(u_{N}\right)\right)-\mu_{1} D\left(z-z\left(u_{N}\right)\right)+\mu_{2}\left(z-z\left(u_{N}\right)\right), z-z\left(u_{N}\right)-Z w\right) \\
=\left(y-y\left(u_{N}\right), z-z\left(u_{N}\right)-Z w\right)+\left(\mu-\mu_{N}, z-z\left(u_{N}\right)-Z w\right) .
\end{gathered}
$$

We can check that $\left(\mu-\mu_{N}, z-z\left(u_{N}\right)-Z w\right)=0$. Then we further derive

$$
\begin{aligned}
& \left((-\Delta)^{\frac{\alpha}{2}}\left(z-z\left(u_{N}\right)\right)-\mu_{1} D\left(z-z\left(u_{N}\right)\right)+\mu_{2}\left(z-z\left(u_{N}\right)\right), z-z\left(u_{N}\right)\right) \\
& =\left((-\Delta)^{\frac{\alpha}{2}}\left(z-z\left(u_{N}\right)\right)-\mu_{1} D\left(z-z\left(u_{N}\right)\right)+\mu_{2}\left(z-z\left(u_{N}\right)\right), Z w\right) \\
& \quad+\left(y-y\left(u_{N}\right), z-z\left(u_{N}\right)-Z w\right) .
\end{aligned}
$$

By integration-by-parts, we have 


$$
\mu_{1}\left(D\left(z-z\left(u_{N}\right)\right),\left(z-z\left(u_{N}\right)\right)\right)=-\mu_{1}\left(z-z\left(u_{N}\right), D\left(z-z\left(u_{N}\right)\right)\right) .
$$

This yields

$$
\mu_{1}\left(D\left(z-z\left(u_{N}\right)\right),\left(z-z\left(u_{N}\right)\right)\right)=0
$$

By (7), we can derive

$$
\begin{aligned}
& \left((-\Delta)^{\frac{\alpha}{2}}\left(z-z\left(u_{N}\right)\right)+\mu_{2}\left(z-z\left(u_{N}\right)\right), z-z\left(u_{N}\right)\right) \\
& \geq \frac{C_{1}}{2}\left|z-z\left(u_{N}\right)\right|_{H^{\alpha / 2}}^{2}+\mu_{2}\left\|z-z\left(u_{N}\right)\right\|^{2}+\frac{C_{1}}{\alpha}\left\|z-z\left(u_{N}\right)\right\|_{\omega^{-\alpha / 2}}^{2} .
\end{aligned}
$$

Note that

$$
\begin{aligned}
& \left((-\Delta)^{\frac{\alpha}{2}}\left(z-z\left(u_{N}\right)\right)-\mu_{1} D\left(z-z\left(u_{N}\right)\right)+\mu_{2}\left(z-z\left(u_{N}\right)\right), Z w\right) \\
& \quad+\left(y-y\left(u_{N}\right), z-z\left(u_{N}\right)-Z w\right) \\
& \leq C\left|z-z\left(u_{N}\right)\right|_{H^{\alpha / 2}}|Z|+C\left\|y-y\left(u_{N}\right)\right\|\left\|z-z\left(u_{N}\right)\right\|+C|Z|\left\|y-y\left(u_{N}\right)\right\| .
\end{aligned}
$$

Then using the Young inequality we further have

$$
\begin{aligned}
\left|z-z\left(u_{N}\right)\right|_{H^{\alpha / 2}}+\left\|z-z\left(u_{N}\right)\right\| & \leq C\left(|Z|+\left\|y-y\left(u_{N}\right)\right\|\right) \\
& \leq C\left(|Z|+\left\|u-u_{N}\right\|\right) .
\end{aligned}
$$

Using (8) and (11) we can get

$$
\begin{aligned}
|Z| & =\left|\frac{1}{|\Omega|} \int_{\Omega}\left(z-z\left(u_{N}\right)\right) d x\right| \\
& \leq C\left(\left\|z\left(u_{N}\right)-z_{N}\right\|+\left\|u-u_{N}\right\|\right) .
\end{aligned}
$$

By (13) we derive

$$
\begin{aligned}
& \left((-\Delta)^{\frac{\alpha}{2}}\left(z\left(u_{N}\right)-z\left(y_{N}\right)\right)-\mu_{1} D\left(z\left(u_{N}\right)-z\left(y_{N}\right)\right)+\mu_{2}\left(z\left(u_{N}\right)-z\left(y_{N}\right)\right), v\right) \\
& =\left(y\left(u_{N}\right)-y_{N}, v\right) .
\end{aligned}
$$

Setting $v=z\left(u_{N}\right)-z\left(y_{N}\right)$ and using Lemma 5 we obtain

$$
\begin{aligned}
& \quad\left|z\left(u_{N}\right)-z\left(y_{N}\right)\right|_{H^{\alpha / 2}}+\left\|z\left(u_{N}\right)-z\left(y_{N}\right)\right\|+\left\|z\left(u_{N}\right)-z\left(y_{N}\right)\right\|_{\omega^{-\alpha / 2}} . \\
& \leq C\left\|y\left(u_{N}\right)-y_{N}\right\|
\end{aligned}
$$

Then using (22) we derive

$$
\begin{aligned}
\left|z-z\left(u_{N}\right)\right|_{H^{\alpha / 2}}+\left\|z-z\left(u_{N}\right)\right\| & \leq C\left(|Z|+\left\|y-y\left(u_{N}\right)\right\|\right) \\
& \leq C\left(\left\|z\left(u_{N}\right)-z_{N}\right\|+\left\|u-u_{N}\right\|\right) \\
& \leq C\left(\left\|z\left(u_{N}\right)-z\left(y_{N}\right)+z\left(y_{N}\right)-z_{N}\right\|+\left\|u-u_{N}\right\|\right) \\
& \leq C\left(\left\|y\left(u_{N}\right)-y_{N}\right\|+\left\|z\left(y_{N}\right)-z_{N}\right\|+\left\|u-u_{N}\right\|\right) \\
& \leq C N^{-s}+C\left\|u-u_{N}\right\| .
\end{aligned}
$$

Inserting above estimate into (19) gives the theorem result.

Note that the estimate of the $\left|\mu-\mu_{N}\right|$ depends on the estimate of the $\left\|u-u_{N}\right\|$. In the following we are going to estimate $\left\|u-u_{N}\right\|$.

Lemma 8. Let $(y, z, u, \mu)$ and $\left(y_{N}, z_{N}, u_{N}, \mu_{N}\right)$ be the solutions of optimality conditions (8) and (11), respectively. Then the following estimates hold

$$
\left\|u-u_{N}\right\| \leq C N^{-s}
$$


Proof. By (8) and (11), we can get

$$
\begin{aligned}
\gamma\left\|u-u_{N}\right\|^{2} & =\int_{\Omega}\left(\gamma u-\gamma u_{N}\right)\left(u-u_{N}\right) d x \\
& =\int_{\Omega}\left(z_{N}-z\right)\left(u-u_{N}\right) d x \\
& =\int_{\Omega}\left(z\left(u_{N}\right)-z\right)\left(u-u_{N}\right) d x+\int_{\Omega}\left(z_{N}-z\left(u_{N}\right)\right)\left(u-u_{N}\right) d x .
\end{aligned}
$$

By (8) and (13), we have

$$
\left((-\Delta)^{\frac{\alpha}{2}}\left(y-y\left(u_{N}\right)\right)+\mu_{1} D\left(y-y\left(u_{N}\right)\right)+\mu_{2}\left(y-y\left(u_{N}\right)\right), v\right)=\left(u-u_{N}, v\right)
$$

and

$$
\begin{gathered}
\left((-\Delta)^{\frac{\alpha}{2}}\left(z-z\left(u_{N}\right)\right)-\mu_{1} D\left(z-z\left(u_{N}\right)\right)+\mu_{2}\left(z-z\left(u_{N}\right)\right), v\right) \\
=\left(y-y\left(u_{N}\right)+\mu-\mu_{N}, v\right) .
\end{gathered}
$$

Then using Green formula and Lemma 3 we have

$$
\begin{aligned}
\int_{\Omega}\left(z\left(u_{N}\right)-z\right)\left(u-u_{N}\right) d x & =\left((-\Delta)^{\frac{\alpha}{2}}\left(y-y\left(u_{N}\right)\right)+\mu_{1} D\left(y-y\left(u_{N}\right)\right)\right. \\
& \left.+\mu_{2}\left(y-y\left(u_{N}\right)\right), z\left(u_{N}\right)-z\right) \\
& =-\left((-\Delta)^{\frac{\alpha}{2}}\left(z-z\left(u_{N}\right)\right)-\mu_{1} D\left(z-z\left(u_{N}\right)\right)\right. \\
& \left.+\mu_{2}\left(z-z\left(u_{N}\right)\right), y-y\left(u_{N}\right)\right) \\
& =-\left(y-y\left(u_{N}\right)+\mu-\mu_{N}, y-y\left(u_{N}\right)\right) .
\end{aligned}
$$

This implies that

$$
\begin{aligned}
& \gamma\left\|u-u_{N}\right\|^{2}+\left\|y-y\left(u_{N}\right)\right\|^{2} \\
= & \left(z_{N}-z\left(u_{N}\right), u-u_{N}\right)+\left(\mu-\mu_{N}, y\left(u_{N}\right)-y\right) \\
= & \left(z_{N}-z\left(u_{N}\right), u-u_{N}\right)+\left(\mu-\mu_{N}, y\left(u_{N}\right)-y_{N}\right)-\left(\mu-\mu_{N}, y-y_{N}\right) .
\end{aligned}
$$

Note that

$$
\mu\left(1, y-y_{N}\right)= \begin{cases}0, & \text { if } \int_{\Omega} y d x<d, \text { then } \mu=0, \\ \geq 0, & \text { if } \int_{\Omega} y d x=d, \text { then } \mu \geq 0, \text { and } \int_{\Omega} y d x \geq \int_{\Omega} y_{N} d x\end{cases}
$$

and

$$
-\mu_{N}\left(1, y-y_{N}\right)= \begin{cases}0, & \text { if } \int_{\Omega} y_{N} d x<d, \text { then } \mu_{N}=0, \\ \geq 0, & \text { if } \int_{\Omega} y_{N} d x=d, \text { then } \mu_{N} \geq 0, \text { and } \int_{\Omega} y d x \leq \int_{\Omega} y_{N} d x .\end{cases}
$$

Then we have $\left(\mu-\mu_{N}, y-y_{N}\right) \geq 0$.

By (14), Lemma 7 and Young inequality we can get

$$
\begin{aligned}
& \gamma\left\|u-u_{N}\right\|^{2}+\left\|y-y\left(u_{N}\right)\right\|^{2} \\
& \leq C\left(\left\|z_{N}-z\left(u_{N}\right)\right\|\left\|u-u_{N}\right\|+\left|\mu-\mu_{N}\right|\left\|y_{N}-y\left(u_{N}\right)\right\|\right) \\
& \leq C\left(\left\|y_{N}-y\left(u_{N}\right)\right\|+\left\|z\left(y_{N}\right)-z_{N}\right\|\right)\left\|u-u_{N}\right\|+C\left(N^{-s}+\left\|u-u_{N}\right\|\right)\left\|y_{N}-y\left(u_{N}\right)\right\| \\
& \leq C\left(\left\|z\left(y_{N}\right)-z_{N}\right\|^{2}+\left\|y_{N}-y\left(u_{N}\right)\right\|^{2}\right)+C N^{-s}\left\|y_{N}-y\left(u_{N}\right)\right\|+\varepsilon\left\|u-u_{N}\right\|^{2} \\
& \leq C N^{-2 s}+\varepsilon\left\|u-u_{N}\right\|^{2} .
\end{aligned}
$$


Here $\varepsilon>0$ is an arbitrary small constant. Further we have

$$
\left\|u-u_{N}\right\| \leq C N^{-s}
$$

Theorem 2. Let $\left(y_{N}, z_{N}, u_{N}, \mu_{N}\right)$ be the solution of (11), and $(y, z, u, \mu)$ be the solutions of (8), respectively. Assume that $\omega^{-\alpha / 2} y, \omega^{-\alpha / 2} z \in B_{\omega^{\alpha / 2}}^{s}$ with $s \geq \alpha / 2$. Then we have

$$
\begin{array}{r}
\left\|y-y_{N}\right\|_{\omega^{-\alpha / 2}}+\left\|z-z_{N}\right\|_{\omega^{-\alpha / 2}}+\left\|u-u_{N}\right\|+\left|\mu-\mu_{N}\right| \leq C N^{-s}, \\
\left\|y-y_{N}\right\|_{H^{\alpha / 2}}+\left\|z-z_{N}\right\|_{H^{\alpha / 2}} \leq C N^{\alpha / 2-s} .
\end{array}
$$

Proof. We conclude from Lemmas 6-8 that

$$
\begin{aligned}
& \left\|y-y_{N}\right\|_{\omega^{-\alpha / 2}}+\left\|z-z_{N}\right\|_{\omega^{-\alpha / 2}}+\left\|u-u_{N}\right\|+\left|\mu-\mu_{N}\right| \\
\leq & C\left(N^{-s}+\left\|u-u_{N}\right\|+\left|\mu-\mu_{N}\right|\right)+C\left(N^{-s}+\left\|u-u_{N}\right\|\right)+C N^{-s} \\
\leq & C N^{-s}
\end{aligned}
$$

and

$$
\begin{aligned}
& \left\|y-y_{N}\right\|_{H^{\alpha / 2}}+\left\|z-z_{N}\right\|_{H^{\alpha / 2}} \\
\leq & C\left(N^{\alpha / 2-s}+\left\|u-u_{N}\right\|+\left|\mu-\mu_{N}\right|\right)+C\left(N^{-s}+\left\|u-u_{N}\right\|\right)+C N^{-s} \\
\leq & C N^{\alpha / 2-s} .
\end{aligned}
$$

\section{Numerical Experiments}

\subsection{Algorithm}

Let

$$
y_{N}=\sum_{l=0}^{N} \hat{y}_{l} \phi_{l}(x), p_{N}=\sum_{l=0}^{N} \hat{p}_{l} \phi_{l}(x) .
$$

Taking the test function $v_{N}=\phi_{k}(x)$ for $k=0,1, \ldots N$, and using Lemmas 1 and 2 yields

$$
\begin{aligned}
& \lambda_{k}^{\alpha} h_{k}^{\alpha / 2} \hat{y}_{k}+\mu_{1} \sum_{l=0}^{N} M_{k, l}^{a} \hat{y}_{l}+\mu_{2} \sum_{l=0}^{N} M_{k, l}^{r} \hat{y}_{l}=\left(f+u_{N}, \phi_{k}\right), \\
& \lambda_{k}^{\alpha} h_{k}^{\alpha / 2} \hat{p}_{k}-\mu_{1} \sum_{l=0}^{N} M_{k, l}^{a} \hat{p}_{l}+\mu_{2} \sum_{l=0}^{N} M_{k, l}^{r} \hat{p}_{l}=\left(y_{N}-y_{d}+\mu_{N}, \phi_{k}\right) .
\end{aligned}
$$

We denote by $M^{d}$ (diffusion term), $M^{a}$ (advection term) and $M^{r}$ (reaction term) the corresponding coefficient matrix. The $k$ th row and $l$ th column entry of matrices $M^{d}, M^{a}$ and $M^{r}$ are calculated as follows

$$
\begin{aligned}
& M_{k, k}^{d}=\lambda_{k}^{\alpha} h_{k}^{\alpha / 2}, \\
& M_{k, l}^{a}=-2(l+1) \int_{-1}^{1} \omega^{\alpha-1}(x) P_{l+1}^{\alpha / 2-1}(x) P_{k}^{\alpha / 2}(x) d x, \\
& M_{k, l}^{r}=\int_{-1}^{1} \omega^{\alpha}(x) P_{l}^{\alpha / 2}(x) P_{k}^{\alpha / 2}(x) d x .
\end{aligned}
$$

Therefore in order to solve the state and adjoint state equation we just need to solve the following equation with different coefficient matrix and right hand terms

$$
\mathcal{A} \hat{\zeta}=\hat{G},
$$


where

$$
\mathcal{A}=M^{d}+\mu_{1} M^{a}+\mu_{2} M^{r} \text {, or } M^{d}-\mu_{1} M^{a}+\mu_{2} M^{r} .
$$

Due to the existence of convection term and reaction term, the coefficient matrix of discrete state equation and adjoint equation are dense. A direct solver will require $O\left(N^{2}\right)$ storage and its complexity is $O\left(N^{3}\right)$. We adopt a matrix-free iterative solver with storage $O(N)$ and computational complexity $O\left(N \log ^{2}(N)\right)$ developed in [35] to solve the above equation.

This iterative solver consists of a fixed-point iteration and fast polynomial transforms. A fixed point iteration is used to solve the state equation in (26)

$$
\hat{\xi}_{k+1}=\hat{\xi}_{k}+\mathcal{P}^{-1}\left(\hat{G}-\mathcal{A} \hat{\xi}_{k}\right) .
$$

Here $\mathcal{P}=M^{d}+\mu_{2} I+\mu_{1} D^{a}$, where $D^{a}$ is a tridiagonal matrix with

$$
D_{k, k+1}^{a}=M_{k, k+1}^{a}, \quad D_{k+1, k}^{a}=M_{k+1, k}^{a}, \quad k=1, \ldots N
$$

the remaining of $D^{a}$ elements are zero. In each iteration, we compute the matrix-vector product $\mathcal{A} \hat{\xi}$ by using fast polynomials transform, instead of forming a matrix. For more details one can refer to [35].

We reduce the cost of the whole algorithm by speeding up the calculation of state equation and adjoint equation. The discrete optimality system can be solved by the following Arrow-Hurwicz algorithm in Algorithm 1 (see [41]).

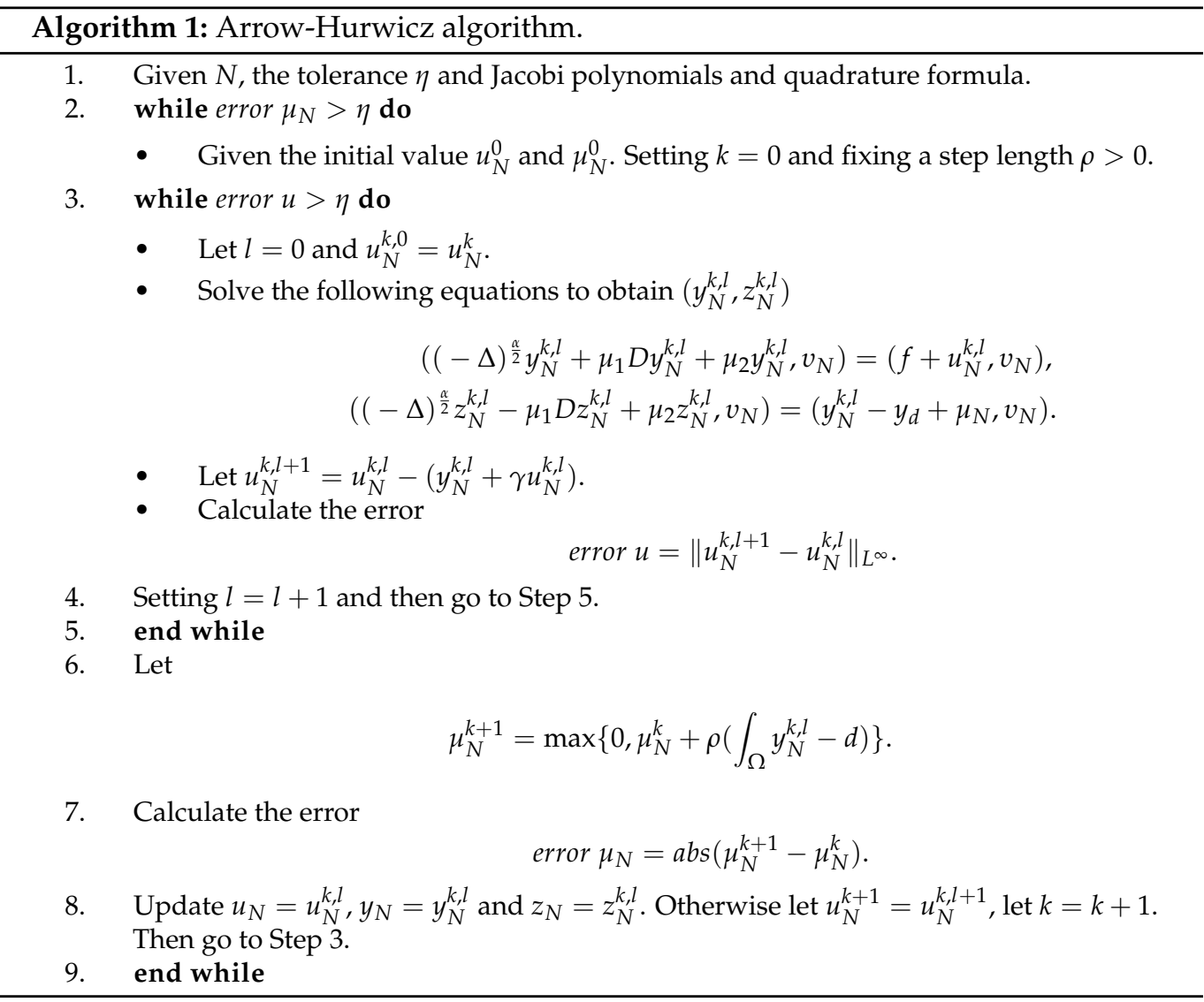




\subsection{Numerical Examples}

Example 1. We consider the optimal control problem (8) with the exact state and adjoint state $y=\left(1-x^{2}\right)^{2}, z=2\left(1-x^{2}\right)^{2}, u=-2\left(1-x^{2}\right)^{2}$. Here $\rho=8, \gamma=1$ and $\mu=0.5$.

$$
\begin{aligned}
f(x) & =\frac{1}{2 \cos (\alpha \pi / 2)}\left[\frac{\Gamma(5)}{\Gamma(5-\alpha)}\left((x+1)^{4-\alpha}+(1-x)^{4-\alpha}\right)\right. \\
& -\frac{4 \Gamma(4)}{\Gamma(4-\alpha)}\left((x+1)^{3-\alpha}+(1-x)^{3-\alpha}\right) \\
& \left.+\frac{4 \Gamma(3)}{\Gamma(3-\alpha)}\left((x+1)^{2-\alpha}+(1-x)^{2-\alpha}\right)\right] \\
& +\mu_{1}(-4) x\left(1-x^{2}\right)+\mu_{2}\left(1-x^{2}\right)^{2}-u, \\
y_{d}(x) \quad= & y-\left\{2 \left\{\frac { 1 } { 2 \operatorname { c o s } ( \alpha \pi / 2 ) } \left[\frac{\Gamma(5)}{\Gamma(5-\alpha)}\left((x+1)^{4-\alpha}+(1-x)^{4-\alpha}\right)\right.\right.\right. \\
& -\frac{4 \Gamma(4)}{\Gamma(4-\alpha)}\left((x+1)^{3-\alpha}+(1-x)^{3-\alpha}\right) \\
& \left.+\frac{4 \Gamma(3)}{\Gamma(3-\alpha)}\left((x+1)^{2-\alpha}+(1-x)^{2-\alpha}\right)\right] \\
& \left.\left.-\mu_{1}(-4) x\left(1-x^{2}\right)+\mu_{2}\left(1-x^{2}\right)^{2}\right\}\right\} .
\end{aligned}
$$

In this case we have $\omega^{-\alpha / 2} y, \omega^{-\alpha / 2} z$ belong to $B_{\omega^{\alpha / 2}}^{5-\frac{\alpha}{2}-\epsilon}$ according to [33]. We numerically demonstrate the results of the convergence in space proved in Theorem 2 . The true and numerical solutions of state variables, adjoint variables and control variables are shown in Figure 1. The numerical experiments results of the convergence order under the weighted $L^{2}$ norm are $5-\frac{\alpha}{2}-\epsilon$. are presented in Tables $1-3$. The convergence order of state variable and adjoint variable in $\|\cdot\|_{H^{\alpha / 2}}$ norm are expected to be $5-\alpha-\epsilon$. The results are shown in Tables 4-6 including different values of $N$ with $\alpha=1.3,1.5,1.7$.

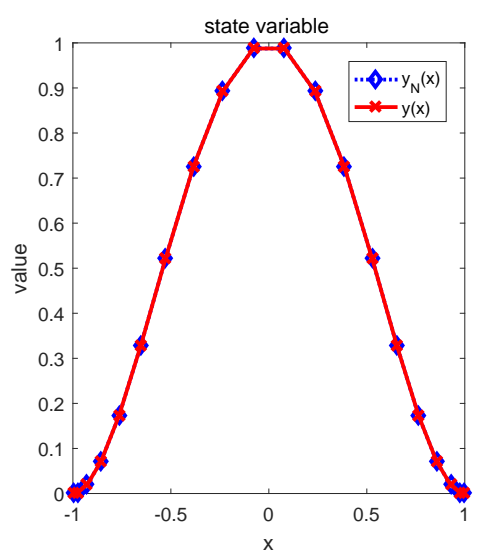

(a)

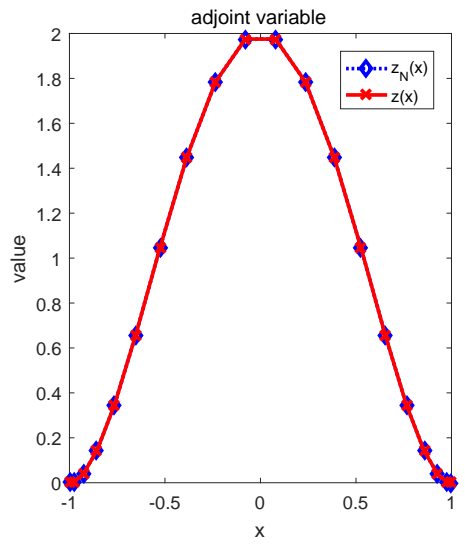

(b)

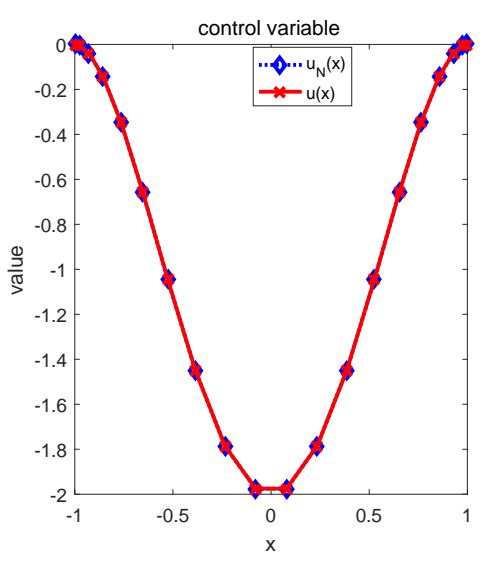

(c)

Figure 1. True solutions and numerical solutions. (left) $y$ and $y_{N}$, (middle) $z$ and $z_{N}$, (right). Here $y_{N}, z_{N}$ and $u_{N}$ are calculated by Algorithm 1. 
Table 1. Errors and convergence rates of $y, z$ in weighted $L^{2}$ norm and $\mu$ with $\alpha=1.30$.

\begin{tabular}{ccccccc}
\hline $\boldsymbol{N}$ & $\left\|y-y_{N}\right\|_{\omega^{-\frac{\alpha}{2}}}$ & Rate & $\left\|z-z_{N}\right\|_{\omega^{-\frac{\alpha}{2}}}$ & Rate & $\left|\boldsymbol{\mu}-\boldsymbol{\mu}_{N}\right|$ & Rate \\
\hline 10 & $5.54 \times 10^{-4}$ & & $1.20 \times 10^{-3}$ & & $2.81 \times 10^{-4}$ & \\
20 & $3.00 \times 10^{-5}$ & 4.21 & $6.24 \times 10^{-5}$ & 4.23 & $1.30 \times 10^{-5}$ & 4.44 \\
40 & $1.58 \times 10^{-6}$ & 4.25 & $3.24 \times 10^{-6}$ & 4.27 & $5.80 \times 10^{-7}$ & 4.48 \\
80 & $8.09 \times 10^{-8}$ & 4.30 & $1.63 \times 10^{-7}$ & 4.32 & $4.42 \times 10^{-8}$ & 3.72 \\
\hline $\mathcal{K}$ & & & $4.35-\epsilon$ & & $4.35-\epsilon$ \\
\hline
\end{tabular}

Table 2. Errors and convergence rates of $y, z$ in weighted $L^{2}$ norm and $\mu$ with $\alpha=1.50$.

\begin{tabular}{ccccccc}
\hline $\boldsymbol{N}$ & $\left\|y-y_{N}\right\|_{\omega^{-\frac{\alpha}{2}}}$ & Rate & $\left\|z-z_{N}\right\|_{\omega^{-\frac{\alpha}{2}}}$ & Rate & $\left|\boldsymbol{\mu}-\mu_{N}\right|$ & Rate \\
\hline 10 & $4.56 \times 10^{-4}$ & & $9.98 \times 10^{-4}$ & & $3.50 \times 10^{-4}$ & \\
20 & $2.63 \times 10^{-5}$ & 4.11 & $5.72 \times 10^{-5}$ & 4.13 & $1.85 \times 10^{-5}$ & 4.24 \\
40 & $1.50 \times 10^{-6}$ & 4.13 & $3.20 \times 10^{-6}$ & 4.16 & $9.12 \times 10^{-7}$ & 4.34 \\
80 & $8.34 \times 10^{-8}$ & 4.17 & $1.75 \times 10^{-7}$ & 4.20 & $4.29 \times 10^{-8}$ & 4.41 \\
\hline $\mathcal{K}$ & & & $4.25-\epsilon$ & & $4.25-\epsilon$ \\
\hline
\end{tabular}

Table 3. Errors and convergence rates of $y, z$ in weighted $L^{2}$ norm and $\mu$ with $\alpha=1.70$.

\begin{tabular}{ccccccc}
\hline$N$ & $\left\|y-y_{N}\right\|_{\omega^{-\frac{\alpha}{2}}}$ & Rate & $\left\|z-z_{N}\right\|_{\omega^{-\frac{\alpha}{2}}}$ & Rate & $\left|\boldsymbol{\mu}-\mu_{N}\right|$ & Rate \\
\hline 10 & $2.71 \times 10^{-4}$ & & $6.77 \times 10^{-4}$ & & $4.11 \times 10^{-4}$ & \\
20 & $1.78 \times 10^{-5}$ & 3.93 & $4.41 \times 10^{-5}$ & 3.94 & $2.54 \times 10^{-5}$ & 4.01 \\
40 & $1.15 \times 10^{-6}$ & 3.94 & $2.76 \times 10^{-6}$ & 4.00 & $1.45 \times 10^{-6}$ & 4.13 \\
80 & $7.19 \times 10^{-8}$ & 4.01 & $1.65 \times 10^{-7}$ & 4.06 & $7.88 \times 10^{-8}$ & 4.20 \\
\hline $\mathcal{K}$ & & & & & & $4.15-\epsilon$ \\
& & & & &
\end{tabular}

Table 4. Errors and convergence rate of $y, z, u$ in non-weighted Sobolev norm with $\alpha=1.30$.

\begin{tabular}{ccccccc}
\hline $\boldsymbol{N}$ & $\left\|\boldsymbol{y}-y_{N}\right\|_{\boldsymbol{H}^{\frac{\alpha}{2}}}$ & Rate & $\left\|z-z_{N}\right\|_{\boldsymbol{H}^{\frac{\alpha}{2}}}$ & Rate & $\left\|\boldsymbol{u}-\boldsymbol{u}_{\boldsymbol{N}}\right\|$ & Rate \\
\hline 10 & $1.50 \times 10^{-3}$ & & $3.10 \times 10^{-3}$ & & $4.55 \times 10^{-4}$ & \\
20 & $1.36 \times 10^{-4}$ & 3.48 & $1.74 \times 10^{-4}$ & 3.51 & $1.92 \times 10^{-5}$ & 4.57 \\
40 & $1.16 \times 10^{-5}$ & 3.55 & $2.32 \times 10^{-5}$ & 3.56 & $7.70 \times 10^{-7}$ & 4.64 \\
80 & $9.48 \times 10^{-7}$ & 3.61 & $1.90 \times 10^{-6}$ & 3.62 & $2.30 \times 10^{-8}$ & 5.06 \\
\hline $\mathcal{K}$ & & $3.70-\epsilon$ & $3.70-\epsilon$ \\
\hline
\end{tabular}

Table 5. Errors and convergence rate of $y, z, u$ in non-weighted Sobolev norm with $\alpha=1.50$.

\begin{tabular}{ccccccc}
\hline $\boldsymbol{N}$ & $\left\|\boldsymbol{y}-\boldsymbol{y}_{\boldsymbol{N}}\right\|_{\boldsymbol{H}^{\frac{\alpha}{2}}}$ & Rate & $\left\|z-z_{N}\right\|_{\boldsymbol{H}^{\frac{\alpha}{2}}}$ & Rate & $\left\|\boldsymbol{u}-\boldsymbol{u}_{\boldsymbol{N}}\right\|$ & Rate \\
\hline 10 & $1.90 \times 10^{-3}$ & & $3.90 \times 10^{-3}$ & & $3.98 \times 10^{-4}$ & \\
20 & $1.94 \times 10^{-4}$ & 3.28 & $3.91 \times 10^{-4}$ & 3.30 & $1.99 \times 10^{-5}$ & 4.32 \\
40 & $1.90 \times 10^{-5}$ & 3.35 & $3.81 \times 10^{-5}$ & 3.36 & $9.59 \times 10^{-7}$ & 4.38 \\
80 & $1.78 \times 10^{-6}$ & 3.42 & $3.56 \times 10^{-6}$ & 3.42 & $4.46 \times 10^{-8}$ & 4.43 \\
\hline $\mathcal{K}$ & $3.50-\epsilon$ & $3.50-\epsilon$ \\
\hline
\end{tabular}

Table 6. Errors and convergence rate of $y, z, u$ in non-weighted Sobolev norm with $\alpha=1.70$.

\begin{tabular}{ccccccc}
\hline $\boldsymbol{N}$ & $\left\|\boldsymbol{y}-\boldsymbol{y}_{\boldsymbol{N}}\right\|_{\boldsymbol{H}^{\frac{\alpha}{2}}}$ & Rate & $\left\|z-z_{N}\right\|_{\boldsymbol{H}^{\frac{\alpha}{2}}}$ & Rate & $\left\|\boldsymbol{u}-\boldsymbol{u}_{\boldsymbol{N}}\right\|$ & Rate \\
\hline 10 & $1.90 \times 10^{-3}$ & & $3.80 \times 10^{-3}$ & & $3.52 \times 10^{-4}$ & \\
20 & $2.21 \times 10^{-4}$ & 3.08 & $4.44 \times 10^{-4}$ & 3.10 & $2.17 \times 10^{-5}$ & 4.02 \\
40 & $2.48 \times 10^{-5}$ & 3.16 & $4.96 \times 10^{-5}$ & 3.16 & $1.24 \times 10^{-6}$ & 4.13 \\
80 & $2.66 \times 10^{-6}$ & 3.22 & $5.32 \times 10^{-6}$ & 3.22 & $6.72 \times 10^{-8}$ & 4.21 \\
\hline $\mathcal{K}$ & & $3.30-\epsilon$ & $3.30-\epsilon$ & \\
\hline
\end{tabular}




\section{Conclusions}

In this paper a spectral Galerkin approximation of fractional advection diffusion optimal control problem with integral state constraint is discussed. Weighted Jacobi polynomials are used to approximate the state and adjoint state. A priori error estimates for state, adjoint state, control variables, and Lagrangian multiplier are derived. Numerical example is presented to verify our theoretical findings. In future, we will further consider optimal control problems with variable order fractional operator.

Author Contributions: F.W. reviewed relevant studies and the literature, accomplished the formula derivation and numerical simulations, and drafted the article. Z.Z. gave the general idea of the study, checked the draft. X.L. provided feasible suggestions and a critical revision of the manuscript. All authors have read and agreed to the published version of the manuscript.

Funding: The work was supported by the NSFC of Shandong Province (No. ZR2016JL004).

Institutional Review Board Statement: Not applicable.

Informed Consent Statement: Not applicable.

Data Availability Statement: The data presented in this study are available on request from the corresponding author.

Acknowledgments: The authors would like to express their most sincere thanks to the referees for their very helpful comments and suggestions, which greatly improved the quality of this paper.

Conflicts of Interest: The authors declare no conflict of interest.

\section{References}

1. Benson, D.A.; Wheatcraft, S.W.; Meerschaeert, M.M. The fractional order governing equations of levy motion. Water Resour. Res. 2000, 36, 1413-1423. [CrossRef]

2. Klafter, J.; Sokolov, I.M. Anomalous diffusion spreads its wings. Phys. World 2005, 18, 29-32. [CrossRef]

3. Meerschaert, M.M.; Sikorskii, A. Stochastic Models for Fractional Calculus; De Gruyter Studies in Mathematics; De Gruyter: Berlin, Germany, 2000; Volume 43.

4. Zhao, D.L.; Liu, Y.S.; Li, X.D. Controllability for a class of semilinear fractional evolution systems via resolvent operators. Commun. Pure Appl. Anal. 2019, 18, 455-478. [CrossRef]

5. Zhao, D.L.; Mao, J. New controllability results of fractional nonlocal semilinear evolution systems with finite delay. Complexity 2020, 2020, 7652648. [CrossRef]

6. Li, X.D.; Caraballo, T.; Rakkiyappan, R.; Han, X.P. On the stability of impulsive functional differential equations with infinite delays. Math. Methods Appl. Sci. 2015, 38, 3130-3140. [CrossRef]

7. Hu, J.T.; Sui, G.X.; Lv, X.X.; Li, X.D. Fixed-time control of delayed neural networks with impulsive perturbations. Nonlinear Anal. Model. Control 2018, 23, 904-920. [CrossRef]

8. Li, X.D.; Yang, X.Y.; Huang, T.W. Persistence of delayed cooperative models: Impulsive control method. Appl. Math. Comput. 2019, 342, 130-146. [CrossRef]

9. Udhayakumar, K.; Rakkiyappan, R.; Li, X.D.; Cao, J. Mutiple psi-type stability of fractional-order quaternion-valued neural networks. Appl. Math. Comput. 2021, 401, 126092.

10. Ionescu, C.; Lopes, A.; Copot, D.; Machado, J.A.T.; Bates, J.H.T. The role of fractional calculus in modeling biological phenomena: A review. Commun. Nonlinear Sci. 2017, 51, 141-159. [CrossRef]

11. Copot, C.; Burlacu, A.; Ionescu, C.M.; Lazar, C.; De Keyser, R. A fractional order control strategy for visual servoing systems. Mechatronics 2013, 23, 848-855. [CrossRef]

12. Muresan, C.I.; Folea, S.; Mois, G.; Dulf, E.H. Development and implementation of an FPGA based fractional order controller for a DC motor. Mechatronics 2013, 23, 798-804. [CrossRef]

13. De Keyser, R.; Muresan, C.I.; Ionescu, C.M. A novel auto-tuning method for fractional order PI/PD controllers. ISA Trans. 2016, 62, 268-275. [CrossRef]

14. Chen, H.Z.; Wang, H. Numerical simulation for conservative fractional diffusion equations by an expanded mixed formulation. J. Comput. Appl. Math. 2016, 296, 480-498. [CrossRef]

15. Kumar, S.; Cao, J.D.; Li, X.D. A numerical method for time-fractional reaction-diffusion and integro reaction-diffusion equation based on quasi-wavelet. Complexity 2020, 2020, 3291723. [CrossRef]

16. Ervin, V.; Roop, J. Variational formulation for the stationary fractional advection dispersion equation. Numer. Methods Partial Differ. Equ. 2006, 22, 558-576. [CrossRef]

17. Jin, B.T.; Lazarov, R.; Pasciak, J.; Rundell, W. Variational formulation of problems involving fractional order differential operators. Math. Comput. 2015, 84, 2665-2700. [CrossRef] 
18. Li, B.J.; Luo, H.; Xie, X.P. Analysis of a time-stepping scheme for time fractional diffusion problems with nonsmooth data. SIAM J. Numer. Anal. 2019, 2, 779-798. [CrossRef]

19. Li, L.Y.; Jiang, Z.W.; Yin, Z. Compact finite-difference method for 2D time-fractional convection-diffusion equation of groundwater pollution problems. Comput. Appl. Math. 2020, 39, 142. [CrossRef]

20. Agrawal, O. A General Formulation and Solution Scheme for Fractional Optimal Control Problems. Nonlinear Dyn. 2004, 38, 323-337. [CrossRef]

21. D'Elia, M.; Glusa, C.; Otarola, E. A priori error estimates for the optimal control of the integral fractional Laplacian. SIAM J. Control Optim. 2019, 57, 2775-2798. [CrossRef]

22. Du, N.; Wang, H.; Liu, W.B. A fast gradient projection method for a constrained fractional optimal control. J. Sci. Comput. 2016, 68, 1-20. [CrossRef]

23. Jin, B.T.; Li, B.Y.; Zhou, Z. Pointwise-in-time error estimates for an optimal control problem with subdiffusion constraint. IMA J. Numer. Anal. 2020, 40, 377-404. [CrossRef]

24. Ye, X.Y.; Xu, C.J. Spectral optimization methods for the time fractional diffusion inverse problem. Numer. Math. Theory Methods Appl. 2013, 6, 499-519.

25. Zaky, M.A.; Tenreiro Machado, J.A. On the formulation and numerical simulation of distributed-order fractional optimal control problems. Commun. Nonlinear Sci. Numer. Simul. 2017, 52, 177-189. [CrossRef]

26. Zhou, Z.J.; Tan, Z.Y. Finite element approximation of optimal control problem governed by space fractional equation. J. Sci. Comput. 2019 78, 1840-1861. [CrossRef]

27. Antil, H.; Otárola, E. A FEM for an optimal control problem of fractional powers of elliptic operators. SIAM J. Control Optim. 2015, 53, 3432-3456. [CrossRef]

28. Baleanu, D.; Defterli, O.; Agrawal, O.P. A Central Difference Numerical Scheme for Fractional Optimal Control Problems. J. Vib. Control 2009, 15, 583-597. [CrossRef]

29. Baleanu, D.; Jajarmi, A.; Sajjadi, S.S.; Mozyrska, D. A new fractional model and optimal control of a tumor-immune surveillance with non-singular derivative operator. Chaos 2019, 29, 083127. [CrossRef]

30. Mohammadi, H.; Kumar, S.; Rezapour, S.; Etemad, S. A theoretical study of the Caputo-Fabrizio fractional modeling for hearing loss due to Mumps virus with optimal control. Chaos Solitons Fractals 2021, 144, 110668. [CrossRef]

31. Ervin, V.; Heuer, N.; Roop, J. Regularity of the solution to 1-D fractional order diffusion equations. Math. Comput. 2018, 87, 2273-2294. [CrossRef]

32. Zhang, Z.Q. Error estimate of spectral galerkin methods for a linear fractional reation-diffusion equation. J. Sci. Comput. 2019, 78, 1087-1110. [CrossRef]

33. Hao, Z.P.; Lin, G.; Zhang, Z.Q. Error estimates of a spectral Petrov-Galerkin method for two-sided fractional reaction-diffusion equations. Appl. Math. Comput. 2020, 374, 125045. [CrossRef]

34. Zhang, L.; Zhou, Z.J. Spectral Galerkin approximation of optimal control problem governed by Riesz fractional differential equation. Appl. Numer. Math. 2019, 143, 247-262. [CrossRef]

35. Wang, F.Y.; Zhang, Z.Q.; Zhou, Z.J. A spectral Galerkin approximation of optimal control problem governed by fractional advection-diffusion-reaction equations. J. Comput. Appl. Math. 2021, 386, 113233. [CrossRef]

36. Babuska, I.; Guo, B.Q. Direct and inverse approximation theorems for the p-version of the finite element method in the framework of weighted Besov spaces Part I: approximability of functions in the weighted Besov spaces. SIAM J. Numer. Anal. 2002, 39, 1512-1538. [CrossRef]

37. Guo, B.Y.; Wang, L.L. Jacobi approximations in non-uniformly Jacobi-weighted Sobolev spaces. J. Approx. Theory 2004, $128,1-41$. [CrossRef]

38. Cacas, E. Pontryagin's principle for state-constrained boundary control problems of semilinear parabolic equations. SIAM J. Control Optim. 1997, 35, 1297-1327. [CrossRef]

39. Casas, E. Error estimates for the numerical approximation of semilinear elliptic control problems with finitely many state constraints. ESAIM Control Optim. Calc. Var. 2002, 8, 345-374. [CrossRef]

40. Hao, Z.P.; Zhang, Z.Q. Optimal regularity and error estimates of a spectral Galerkin method for fractional advection-diffusionreaction equations. SIAM J. Numer. Anal. 2020, 58, 211-233. [CrossRef]

41. Niu, H.F.; Yang, D.P. Finite element analysis of optimal control problem governed by stokes equations with L2-norm stateconstraints. J. Comput. Appl. Math. 2011, 29, 589-604. 